\title{
Characterization and pathogenicity of fowl adenovirus serotype 4 isolated from eastern China
}

\author{
Kai Wang ${ }^{1+}$, Haiwei Sun ${ }^{1+}$, Yunzhang $\mathrm{Li}^{1}$, Zhiwei Yang ${ }^{1}$, Jianqiang Ye ${ }^{2}$ and Hongjun Chen ${ }^{1 *}$ (D)
}

\begin{abstract}
Background: Fowl adenovirus outbreaks have occurred in China since June 2015. This virus is an emerging infectious disease that causes hydropericardium syndrome and inclusion body hepatitis (HPS-IBH), resulting in significant economic loss to poultry farmers. Five fowl adenovirus (FAdV) strains ( $H N, A Q, A H 726, J S 07$ and $A H 712)$ were isolated from Jiangsu and Anhui provinces.

Results: Phylogenetic analysis revealed that the five isolates belonged to species C fowl adenovirus serotype 4 . An 11 amino-acid deletion in ORF29, relative to an older viral isolate, JSJ13, was observed for all five strains described here. In chicken experiments, 80-100\% birds died after intramuscular inoculation and displayed lesions characteristic of HPS-IBH. The viral DNA copies were further detected by hexon-probe based real-time polymerase chain reaction $(P C R)$ in the chicken samples. The viral loads and cytokine profiles were recorded in all the organs after infections. Despite minor genetic differences, the 5 strains displayed significantly different tissue tropisms and cytokine profiles.

Conclusions: Our data enhance the current understanding some of the factors involved in the pathogenicity and genetic diversity of the FAdV serotype 4 (FAdV-4) in China. Our work provides theoretical support for the prevention and control of HPS-IBH in chickens.
\end{abstract}

Keywords: Fowl adenovirus serotype 4, Hydropericardium syndrome, Inclusion body hepatitis, Pathogenesis

\section{Background}

Fowl aviadenoviruses (FAdVs) are classified into five species (A-E) and 12 serotypes (FAdV-1 to $8 \mathrm{a}$ and $-8 \mathrm{~b}$ to 11 ) $[1,2]$. FAdVs infects mostly 3-6-weeks-old broilers [3, 4], and is readily transmitted both horizontally by the fecal-oral route and vertically by embryonated eggs $[5,6]$. Serotype 4 Fowl adenovirus (FAdV-4) belongs to group I adenoviruses in the genus Aviadenovirus, also known as 'Angara Disease', was first reported in Angara Goth, Pakistan in 1987. FAdV4 causes Hydropericardium syndrome (HPS), Inclusion body hepatitis (IBH), respiratory tract disease, and/or gizzard erosion in chickens [7-9].

Since mid-2015, outbreaks of novel genotype FAdV-4 infections in China and Korea have caused great economic losses in the poultry industry, leading to high mortality, characterized by congestive kidney, pericardial

\footnotetext{
* Correspondence: vetchj@shvri.ac.cn

${ }^{\dagger}$ Kai Wang and Haiwei Sun contributed equally to this work.

'Shanghai Veterinary Research Institute, CAAS, Shanghai 200241, China

Full list of author information is available at the end of the article
}

effusion, hyperemic and enlarged liver with petechial hemorrhages and punctiform areas in broilers [10], commercial chickens [11], and ducks [12].

Recent Chinese FAdV-4 isolates have significant deletions in their genome compared with FAdV serotype 4 strains from other countries [13, 14]. However, the molecular mechanisms underlying the infection and pathogenesis of FAdV-4 remain unclear.

In the present study, several strains of FAdV-4 were isolated from Eastern China, their genomes were sequenced, and virulence assays were conducted in chickens.

\section{Results \\ Identification and characterization of FAdV-4 variant isolates}

Chicken liver samples containing lesions indicative of inclusion body hepatitis-pericarditis syndrome were inoculated into SPF eggs. The embryos died after 3-7 days, showing classical lesions of fowl adenovirus infection with dysplasia, 
surface flushing, and bleeding. The livers of the dead embryonated eggs contained yellow-brown blood and necrotic spots. Allantoic fluids were collected and used for total viral DNA extraction, followed by PCR amplification of a conserved region of the hexon gene. Five viral isolates were identified as FAdVs and designated as $\mathrm{HN}, \mathrm{AQ}$, JS07, AH712, and $\mathrm{AH} 726$, respectively (the isolation of JS07 has been previously described by Wang et al., 2016 [15]. The samples were passaged in 7 day- old SPF chicken embryonated eggs and then purified in primary chicken embryo kidney (CEK) cells by plaque assay after typical CPE formation occurred (Fig. 1a). Virus titers in the infected embryos and CEK cells were $10^{8}$ to $10^{8.5} \mathrm{TCID}_{50} / \mathrm{ml}$. Viral particles were observed to be regular hexahedrons using electron microscopy. The diameter of the viral particle was approximately $80-100 \mathrm{~nm}$, which appeared as crystals arranged in the cytoplasm of CEK cells consistent with the characteristics of fowl adenovirus (Fig. 1b).

\section{Analysis of complete sequences of FAdV-4 isolates}

To investigate the molecular pathogenicity of the isolates, the viral genomes were first sequenced. The genome of isolate AH712 was 43,725 base pairs (bp) in length, and the other four strains were $43,723 \mathrm{bp}$. The whole genome nucleotide sequences of the isolates were deposited in GenBank (Table 1). The strains belong to species C FAdV serotype 4 (Fig. 2a). Compared with ON1 (GU188428), a natural deletion of $1966 \mathrm{bp}$ was observed at the position of nt 35,425 based on ON1's genome. This deletion includes two open reading frames, ORF19 and ORF29 (Fig. 2c), which were also present in the recent Chinese variants [16]. The 5 strains are similar to the recently reported highly virulent HLJ/15118 strain, and the non-pathogenic strains clustered into a subgroup. Based on the alignment of the genome sequences of all FAdV-4 strains by the ClustalW method, the strains were divided into two genotypes. All the recent virulent strains were located in the genotype 2 of FAdV-4 (Fig. 2b), whereas three nonpathogenic strains, B1-7, ON1 and KR5 belong to genotype 1. Compared with the Chinese strain JSJ13 isolated in 2013, there were $33 \mathrm{nt}$ deletions in the ORF29 sequence of the five strains. Compared with the classical non-pathogenic strain ON1, there were different levels of GAGA motif repeats in the isolates at 19530-19551 nt (Fig. 2c). The mutations were dispersed throughout the genes encoding the ORF14A, pTP, $52 \mathrm{~K}$, and $100 \mathrm{~K}$ proteins (Fig. 2c). Comparison of variable amino acid sequences from Fiber-2 (FAdV surface Fiber protein 2) among HPS and non-HPS isolates are also shown in (Additional file 1: Table S3).

\section{Quantification of FAdV-4 virus particles by TaqMan probe real-time PCR}

We developed a TaqMan probe fluorescence quantitative polymerase chain reaction (qPCR) method to rapidly

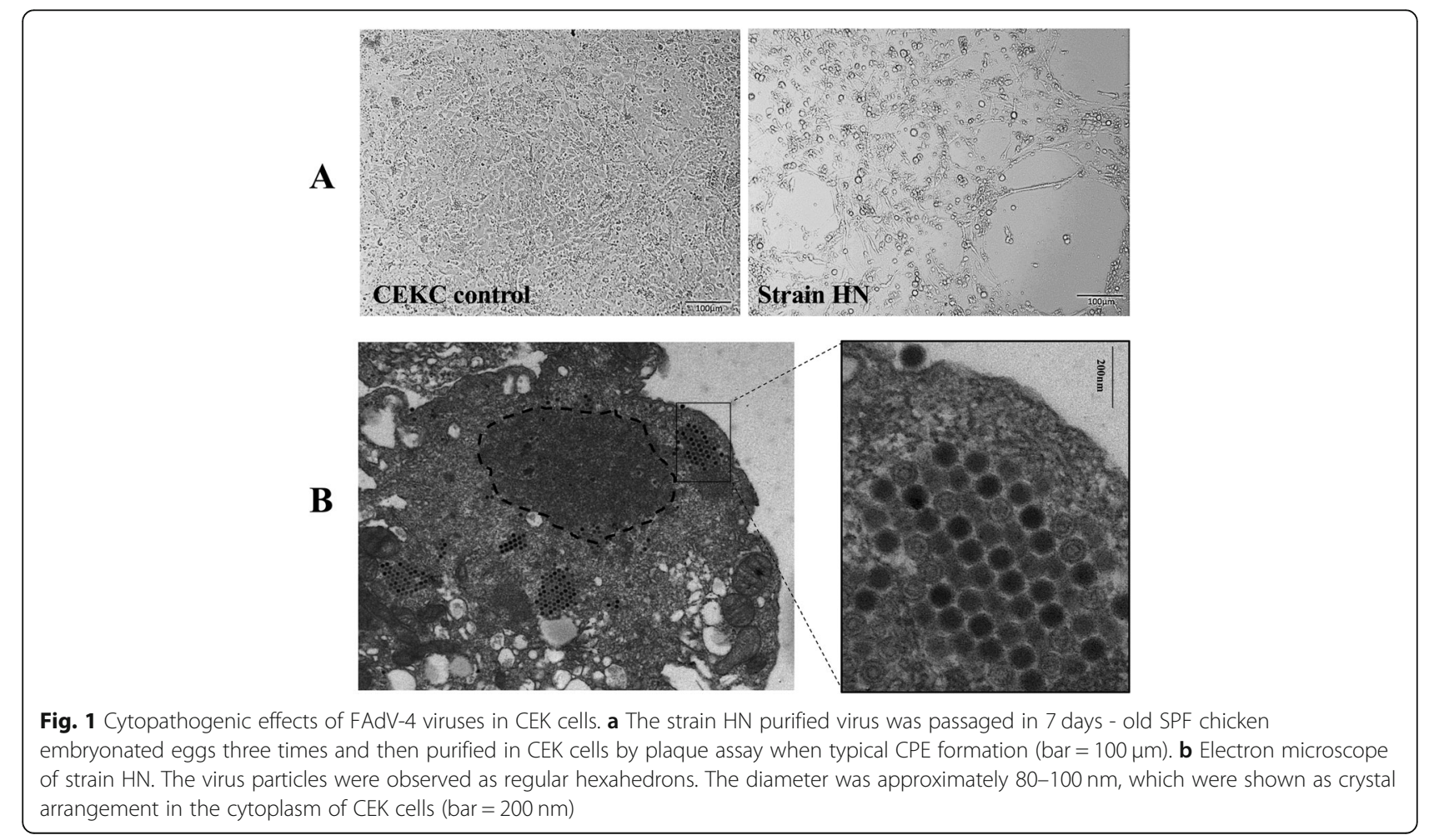


Table 1 The data of compete sequences of FAdV-4 isolates in unvaccinated chickens

\begin{tabular}{|c|c|c|c|c|c|c|}
\hline Strain & Location & Chicken type & Collection date & Size $(k b)$ & Accession Number & Titers $\left(\mathrm{TCID}_{50} / \mathrm{mL}\right)$ \\
\hline $\mathrm{JSO7}$ & Jiangsu & Broiler & 2015.11 .2 & 43,723 & KY436519 & $2.32 \times 10^{7}$ \\
\hline $\mathrm{AQ}$ & Anhui & San-Huang Layer & 2016.6.28 & 43,723 & KY436520 & $5.00 \times 10^{8}$ \\
\hline $\mathrm{HN}$ & Anhui & San-Huang Layer & 2016.6 .28 & 43,723 & KY379035 & $2.81 \times 10^{8}$ \\
\hline $\mathrm{AH} 712$ & Anhui & 817 broiler & 2016.7.12 & 43,725 & KY436522 & $1.08 \times 10^{8}$ \\
\hline $\mathrm{AH} 726$ & Anhui & San-Huang Layer & 2016.7.26 & 43,723 & KY436521 & $1.58 \times 10^{8}$ \\
\hline
\end{tabular}

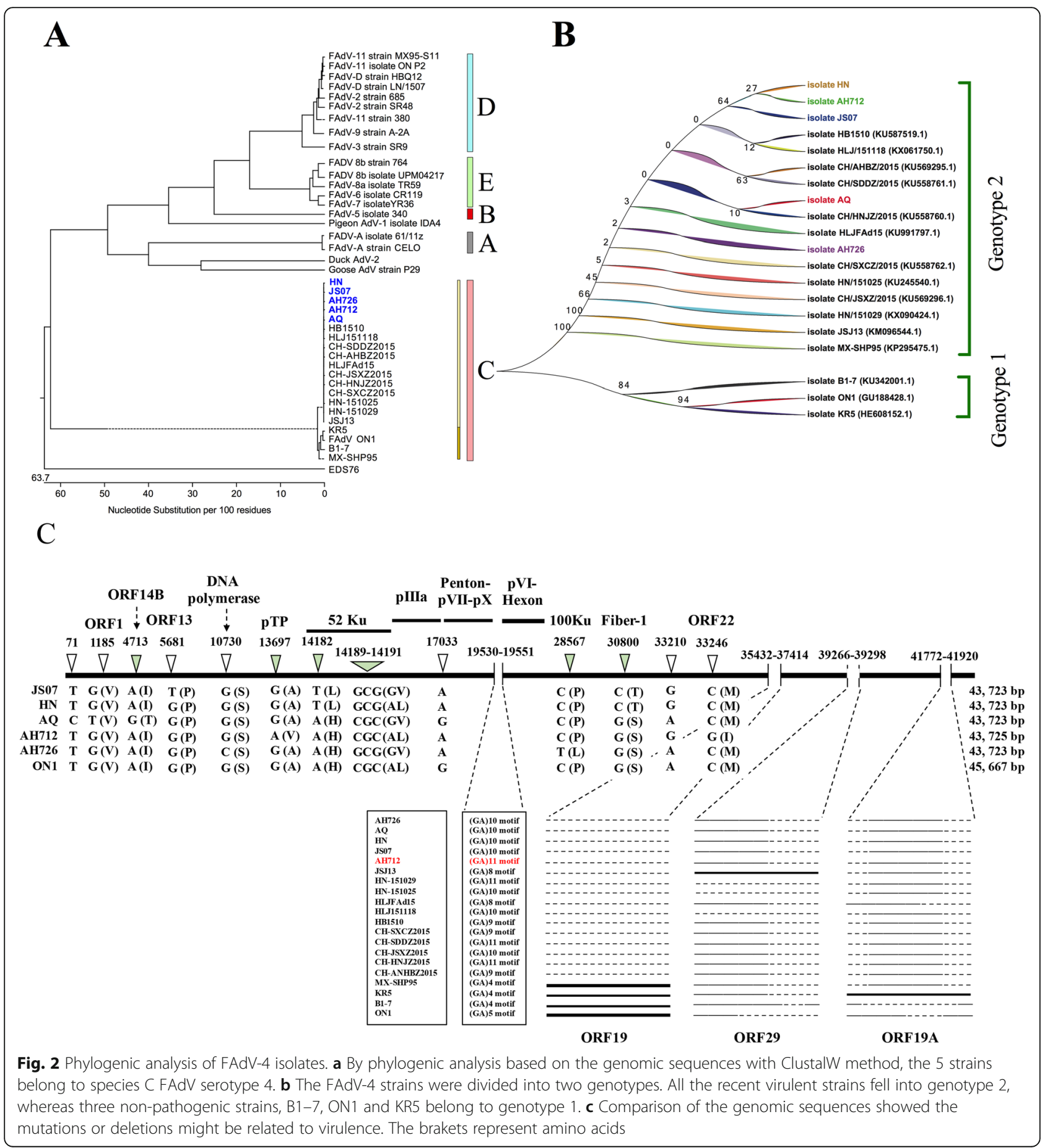


and accurately detect and quantify FAdV-4. We used a pGEM-T easy-hexon plasmid to generate the FAdV-4 DNA standard curve. The standard linear equation is $y=-3.52 x+35.98\left(R^{2}=0.998\right)$. The lowest limit of detection was 22.8 copies/ $\mu$ l, which is 100 times higher than the conventional PCR method. The method laid the foundation for further detection of FAdV-4 isolate in vitro and in vivo, as well as a tool for rapid clinical diagnosis of the disease.

\section{Pathogenicity of recent strains in SPF chickens}

All dead chickens in the infected groups showed severe HPS and IBH (Fig. 3a), with yellow and hemorrhagic livers and pericardial effusion (Fig. 3a). Tissue samples were collected and stored at $-80{ }^{\circ} \mathrm{C}$ for titration or in $10 \%$ neutral formalin for Hematoxylin \& Eeosin ( $\mathrm{H} \& \mathrm{E})$ staining. In the infection experiment, the lethality rates of $\mathrm{HN}, \mathrm{AQ}$, and AH712 were $100 \%$, and the lethality rates of JS07 and AH726 were $80 \%$ (Fig. 3b). Viral genome copy numbers in heart, liver, spleen, lung, brain, trachea, glandular stomach, duodenum, jejunum, cecum, rectum, air sac, bursa of Fabricius, pancreas, and thymus samples were determined using TaqMan probe qPCR and are summarized in Fig. 4. High viral loads were detected in the oralpharyngeal and cloacal swabs of infected chickens. All the isolates caused symptoms consistent with acute fowl adenovirus infection within 3 dpc. At $3 \mathrm{dpc}$, the mean titers were $3 \times 10^{5}$ DNA copies/ml in oral swabs and $1 \times 10^{6}$ DNA copies $/ \mathrm{ml}$ in cloaca swabs from the $\mathrm{HN}$ strain challenge group (Fig. 4a). High viral titers were observed in most organs, with livers having the highest viral loads, followed by the duodenum, jejunum, caecum, rectum, and finally the brain (Fig. 4b). No viral DNA was detected in chickens before inoculation or in mockinfected chickens. Infected chicken livers presented with typical basophilic inclusions, a large number of inflammatory cells infiltrated into the kidneys slices, and the infected bursa of Fabricius had structural disorders and reduced numbers of lymphocytes. The liver, kidney and bursa of Fabricius reacted with mAb 1B4 against Hexon, further indicating that presence of virus in these tissues (Fig. 5).

High expression of cytokine genes during acute infection Based on the calculated lethality values, strains $\mathrm{HN}$ and AH726 were selected for further analyses. Cytokine gene expression following infection was measured in the liver, kidney and bursa of Fabricius. The expression levels of $I L$ $1 \beta, I F N-\alpha, I F N-\beta, I F N-\gamma$, and $I L-10$ mRNAs were shown in Fig. 6. Compared to the PBS control group, there was a statistically significant increase in $I L-1 \beta, I F N-\beta$, and $I F N-\gamma$ transcriptional levels of the kidney and bursa of Fabricius samples in the infected chickens at 3 d.p.i $(p<0.01)$. However, in liver only $I L-1 \beta$ mRNA level was significantly higher in the infected group at 3 d.p.i $(p<$ $0.01)$. Compared to the high virulence of strain $\mathrm{HN}$ with $100 \%$ mortality, the $I L-1 \beta, I F N-\alpha, I F N-\beta, I F N-\gamma$, and $I L-10$ mRNA levels in bursa of Fabricius or kidney samples of the virulent strain AH726 with $80 \%$ mortality were 1.7-5.4 folds less than the levels of strain $\mathrm{HN}$, respectively $(p<0.05)$.

\section{Discussion}

Since 2015, a highly contagious disease with severe hydropericardium syndrome- inclusion body hepatitis (HPS-IBH) has spread in large-scale broiler farms in China $[6,17]$. HPS-IBH disease results in a huge economic loss through caused a large number of chicken's death [16]. In our study, five hypervirulent strains were isolated from several chicken farms in Eastern China and characterized to be serotype 4, based on phylogenetic analysis of the full length of genomic sequences. The whole genome sequences of five highly pathogenic FAdV-4 isolates have enhanced the current understanding of the pathogenesis of FAdVs. Meanwhile, the size of five FAdV-4 isolates in China was significantly different from that reported previously [18]. Alignment of the nucleotide sequence revealed a deletion in ORF19 and ORF29 (Fig. 2). However, the effects of the deletion and sequence differences in ORF19 and ORF29 on viral replication and pathogenicity remain to be studied.

To characterize the virulence of the new isolates, the purified strains were used to challenge SPF chickens and were found to cause $80 \%$ (strains AH726 and JS07) to $100 \%$ (strains HN, AQ and AH712) mortality. Dead chickens showed severe HPS-IBH, which was almost identical to those of naturally infected chickens (Figs.3, 4 and 5). The fact that these strains contain a truncated ORF29 gene and are highly pathogenic suggests that the deletion of ORF29 gene might influence the virulence of the novel Chinese FAdV-4 isolates.

Comparison with all the genomes in Genbank database showed minor differences in the pTP, $52 \mathrm{~K}$, and $100 \mathrm{~K}$ proteins (Fig. 2). Cytokine gene expression associated with $\mathrm{T}$ helper 1 (Th1), pro-inflammatory, and immuneregulatory activities, namely IL- $1 \beta$, IFN $-\alpha$, IFN- $\beta$, IFN- $\gamma$, and IL-10, were investigated in the liver, bursa, and kidney (Fig. 6). The mRNA expression levels of proinflammatory cytokines (IL-6 and IL-8) and interferon-stimulated genes (Mx and OAS) were significantly upregulated by a recent virulent isolate SD0828 in chickens and tissue cultures [19]. However, most of the cytokines showed slight upregulation in the chickens without remarkable changes. The FAdV-4 avirulent strain ON1 (accession No. GU188428) induced neutralizing antibodies as well as the expression 

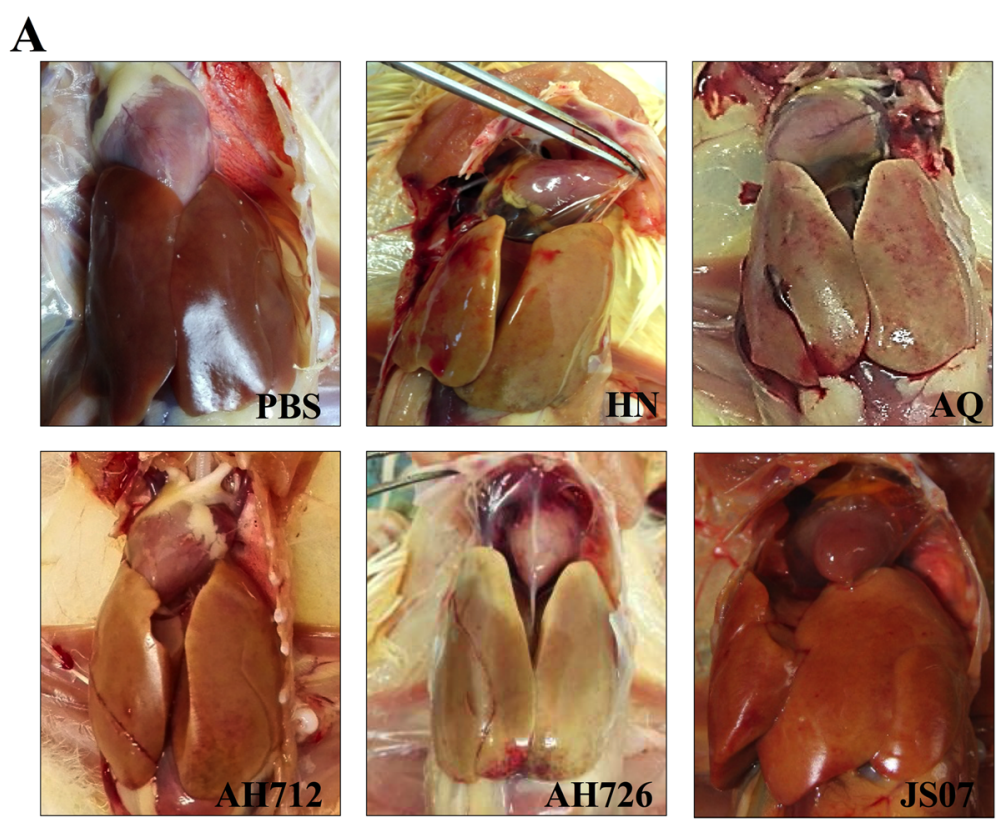

B

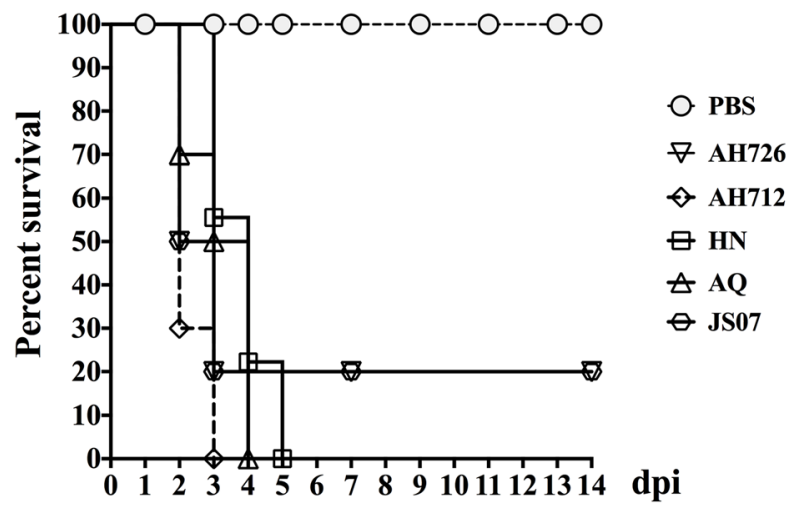

Fig. 3 Pathogenicity of the recent strains in 3-week-old SPF chickens. a Gross lesions of FAdV-4 strains. All dead chickens in the infected groups showed severe HPS-IBH syndrome. $\mathbf{b}$ The survival rate of the isolates

of cytokines, such as IFN- $\gamma$ and IL-10 in the liver [20]. In the current study, cytokine expression was monitored following FAdV-4 infection (Fig. 5) and a remarkable increase in pro-inflammatory cytokine expression was observed. IFN- $\alpha$, IFN- $\beta$, and IFN- $\gamma$ are critical for host defense against a variety of pathogens [20-22]. IL-10 is a pleiotropic cytokine that has both immunosuppressive and immunostimulatory effects on many cell types [20]. Studies have shown in chickens and mammals IL-10 can inhibits Th1 responses [23], thus the $\mathrm{T}$ cell activity is inhibited. In the current study, IL-10 in the liver was significantly up-regulated at 3 d.p.i. in the infected group. Our finding is in agreement with previous reports [20], and may indicate an immune evasion mechanism used by the virus to support persistence.

In summary, the recently outbreak of HPS-IBH in eastern China was associated with a new type of hypervirulent
FAdV serotype 4 variant, and the mortality was much higher than previously reported mortality. The chicken infection model established in this study will be important for the evaluation of antiviral therapy for FADV infection.

\section{Conclusions}

These data will help us to better understand the molecular epidemiology and genetic diversity of the currently circulating FAdV-4 strains in Eastern China. Meanwhile, and provide new ideas for studying the molecular mechanism of FAdV-4 pathogenicity and immune evasion.

\section{Methods}

Sampling and virus amplification in specific pathogen free (SPF) embryonated eggs

In this study, chicken liver samples were collected and isolated from a San-Huang chicken layer farm with IBH 


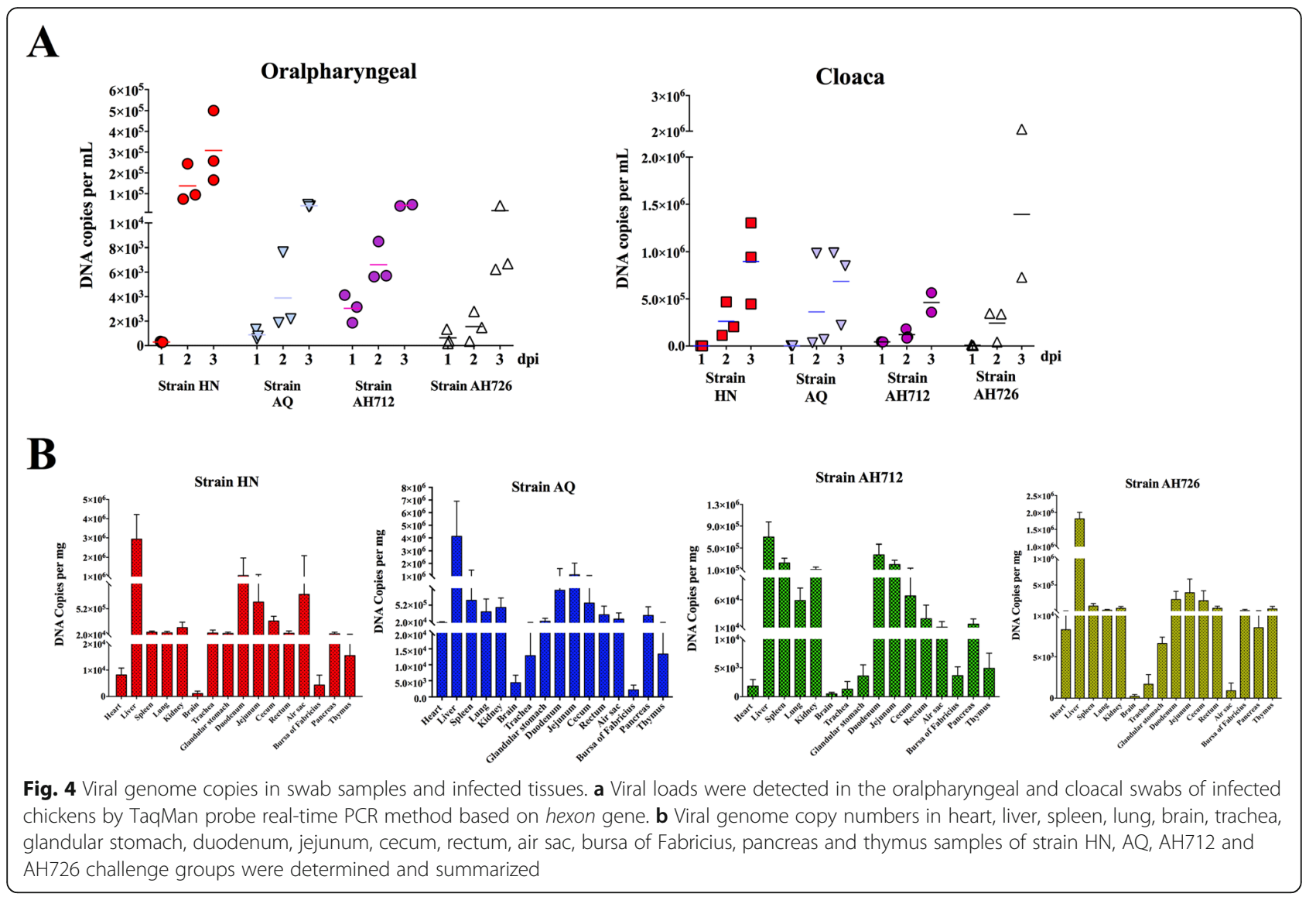

and HPS in three provinces (Jiangsu, Shandong, and Anhui) between December 2015 and December 2016. Then, the liver samples were homogenized in $10 \%$ (wt/vol) in $0.01 \mathrm{M}$ phosphate-buffered saline $(\mathrm{pH} 7.4)$ containing an antibiotic-antimycotic solution (Gibco BRL, Grand Island, NY). The homogenates were centrifuged at 10, $000 \times \mathrm{g}$ for $10 \mathrm{~min}$ after three freeze-thaw cycles. The supernatants were passed through $0.45-\mu \mathrm{m}$ filters. The homogenates were inoculated into 7-day embryonated chicken eggs and then the embryonated eggs were incubated for 7 days, which were obtained from Merialvital Co. (Beijing, China). The allantoic fluids were collected in vials. The viruses were purified by end-point dilution for three times in SPF eggs. The viral titers were determined and calculated according to the Reed-Muench method for $50 \%$ egg lethal dose $\left(E^{2} D_{50}\right)$ following the protocol from a previous study [24].

\section{Purification in CEK cells by plaque assay}

Viruses were amplified and purified in CEK cells [25]. Briefly, primary CEK cells were prepared from the kidneys of 16-18 days-old SPF chicken embryos (SPF chicken embryos were obtained from Beijing Merial Vital Laboratory Animal Technology Co., Ltd., Beijing,
China) and maintained in Dulbecco's Modified Eagle Medium (DMEM) (Gibco) supplemented with 10\% fetal bovine serum. The allantoic fluids containing the isolates were used to infect CEK cells at a confluency of $90 \%$ and the culture medium was replaced with DMEM supplemented with $2 \%$ fetal bovine serum (Gibco). The infected CEK cells were maintained at $37^{\circ} \mathrm{C}$ and $5 \% \mathrm{CO}_{2}$, and the supernatants were harvested at $72 \mathrm{~h}$ postinoculation (hpi) when characteristic cytopathic effects (CPE) were observed. The isolates were then purified three times by plaque assay and subsequently amplified in CEK cells. The stock virus of each generation was stored at $-80^{\circ} \mathrm{C}$ for titration.

\section{Determination of $50 \%$ tissue culture infection doses $\left(\mathrm{TCID}_{50}\right)$}

The $\mathrm{TCID}_{50}$ values of the isolates were determined using 96-well plates (Thermo Scientific, USA) coated with Leghorn male hepatoma chicken liver (LMH) cells (ATCC CRL-2117). LMH cells were infected with tenfold dilutions of virus stocks from $10^{-1}$ to $10^{-10}$ in triplicate for each dilution and three wells for the negative control. The plates were incubated at $37^{\circ} \mathrm{C}$ and plaques were observed by microscope on a daily basis. After 5 


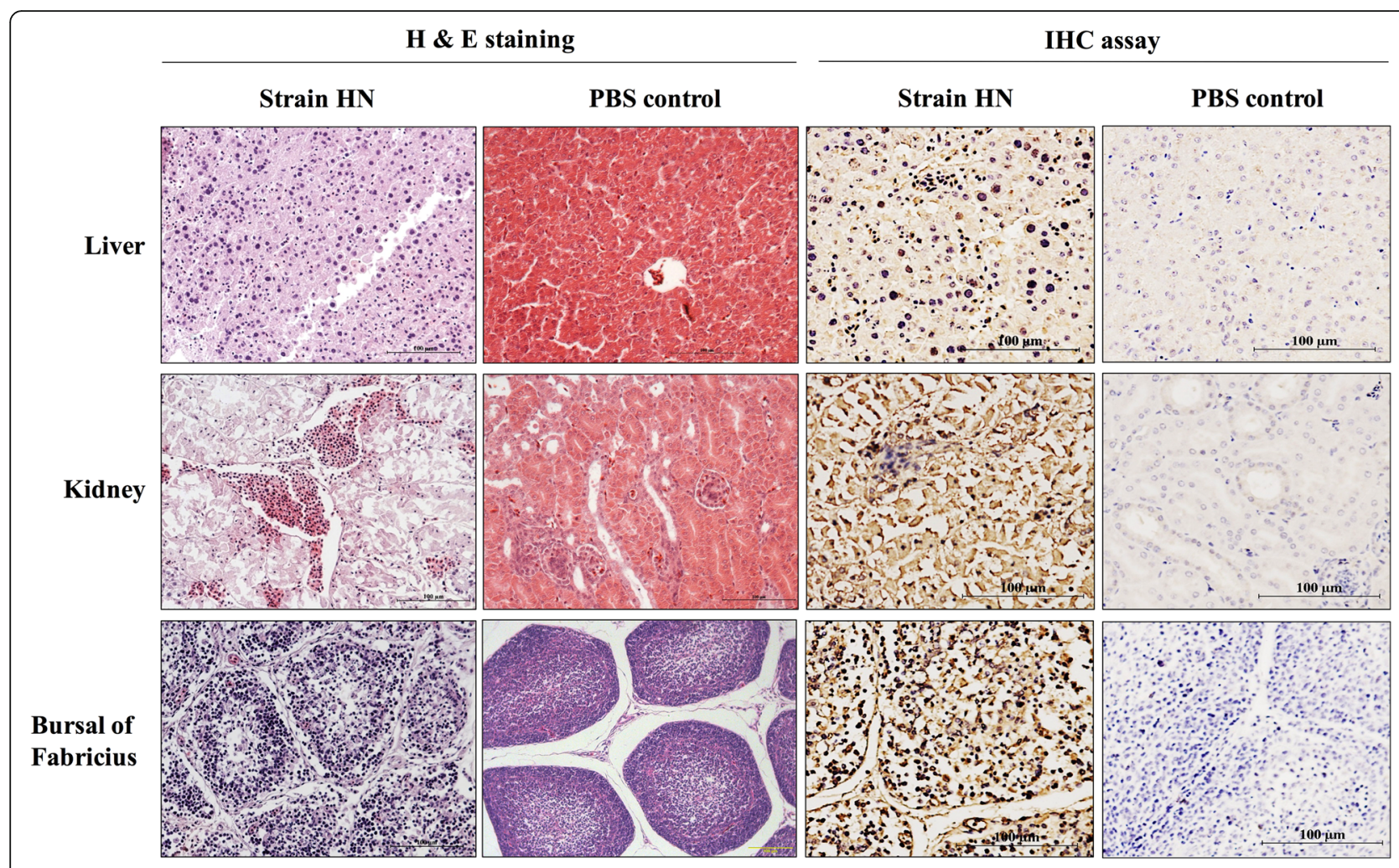

Fig. $5 \mathrm{H}$ \& E staining and immunohistochemical $(\mathrm{IHC})$ observation. The slices of strain $\mathrm{HN}$ - infected liver, kidney, and the bursa of Fabricius were observed by $\mathrm{H} \& \mathrm{E}$ staining and reacted with mAb 1B4 against Hexon by $\mathrm{IHC}$ assay. Magnification is $\times 20$

days of incubation, the $\mathrm{TCID}_{50}$ value was determined according to the Reed and Muench method [24].

Polymerase chain reaction (PCR) and genome sequencing The isolates were identified as FAdV-positive by PCR with primers hexon-852F and hexon-1518R. The primers, highly conserved among all the FAdV isolates, were used to amplify a 667-bp fragment in the hexon gene (from position 852 to 1518 based on the hexon gene of FAdV4 strain ON1). For full-length sequencing, primers were designed. Sequences are shown in Additional file 1: Table S1. All the PCR products were generated with the following protocol: $94^{\circ} \mathrm{C}$ for $5 \mathrm{~min}$ followed by 30 cycles of $94{ }^{\circ} \mathrm{C}$ for $30 \mathrm{~s}, 55^{\circ} \mathrm{C}$ for $1 \mathrm{~min}, 72^{\circ} \mathrm{C}$ for $3 \mathrm{~min}$ and then $72^{\circ} \mathrm{C}$ for $10 \mathrm{~min}$. The PCR products were gelextracted and sequenced using Sanger method by Genewiz (Suzhou, China). The nucleotide sequences of the FAdV isolates were assembled and aligned with homologous sequences using the Lasergene 10 sequence analysis software package (DNAStar, Madison, WI). The phylogenetic tree for the complete genomes was constructed by MEGA6 using a maximum-likelihood method with 1000 bootstrap replicates. The schematic representations of the complete genome sequences were drawn by SnapGene software (GSL Biotech, Chicago, IL).

\section{Transmission electron microscopy}

The primary CEK cells were seeded on coverslips and infected with 10 m.o.i. strain $\mathrm{HN}$ virus for $48 \mathrm{~h}$, then the supernatants were removed and the cells were washed thrice with PBS. The infected CEK cells were then scraped and centrifuged in PBS buffer, discard the supernatants and fixed in phosphate buffered $2.5 \%$ glutaraldehyde buffer for $1 \mathrm{~h}$. The virus pellets were then negatively stained with $1 \%$ phosphotungstic acid (Sigma, USA) for $30 \mathrm{~s}$ and mounted onto one-slot formvar nickel grids. The samples on grids were observed by transmission electron microscope (Tecnai G2 Spirit BIOTWIN, Netherlands).

\section{Pathogenesis in SPF chickens}

To determine the pathogenicity of the isolates, animal experiments were performed using three-week-old SPF White Leghorn chickens (9 chickens/group, obtained from Merial Vital Laboratory Animal Technologies Co., LTD, Beijing, China.) challenged intramuscularly (I. M) with the isolates at a dose of $1 \times 10^{6} \mathrm{TCID}_{50} / 100 \mu \mathrm{l}$. The negative control was challenged with $100 \mu \mathrm{l}$ sterile PBS. At 1,2 , and 3 days post-challenge $(\mathrm{dpc})$, the oralpharyngeal and cloacal swabs were collected and determined for virus shedding. After $3 \mathrm{dpc}$, three chickens in each group were euthanized using carbon dioxide, and organs 


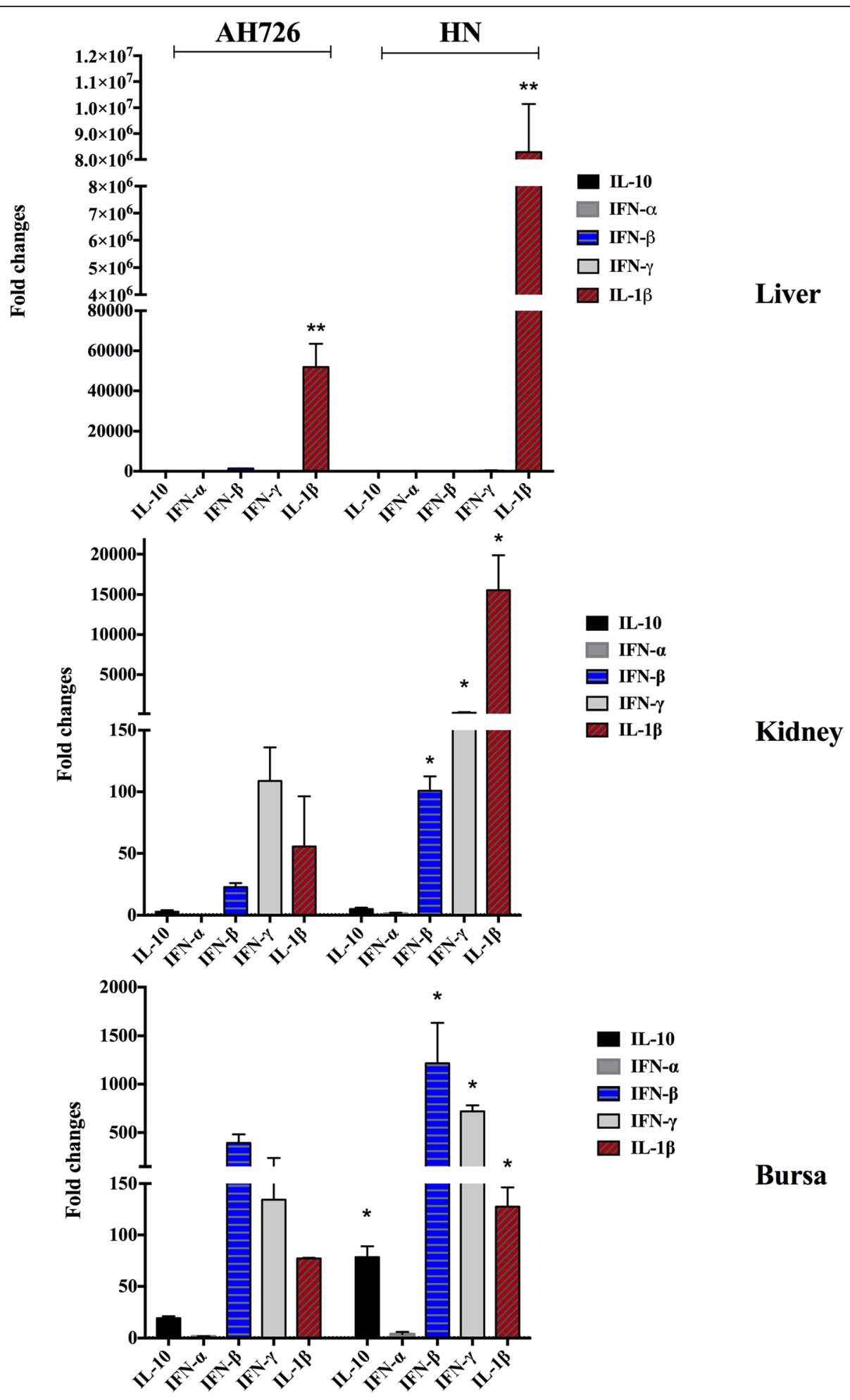

Fig. 6 The changes of cytokines in the infected tissues. Expression of mRNA levels of cytokine genes after I. M inoculation of chickens with strains AH726 and HN were measured compared to the data of the mock as control. The fold changes on the mRNA transcriptional levels of IL-1 $\beta$, IFN- $a$, IFN- $\beta$, IFN- $\gamma$, and IL-10 were illustrated and calculated with the $2^{-\Delta \Delta C t}$ method. Note: statistical significance as $p<0.05\left(^{*}\right)$ or $p<0.01\left(^{* *}\right)$ 
were collected and fixed in either 10\% neutral formalin buffer ( $\mathrm{pH}$ 7.2) or at $-80^{\circ} \mathrm{C}$ for RNA extraction. The survival of the remaining chickens was monitored for 14 days and then euthanized by carbon dioxide.

\section{Quantification of viral load in tissues}

Total DNA was extracted with DNeasy ${ }^{\circ}$ tissue kit (Qiagen Inc., Gaithersburg, MD) and the viral load in the tissues was determined using a TaqMan probe fluorescence quantitative polymerase chain reaction (qPCR) method. The FAdV-4 hexon gene was used as an indicator for the presence of viral DNA. The forward and reverse primers were hexon-1293F and hexon-1417R, respectively. The TaqMan probe was hexon-Probe (Additional file 1: Table S2). To generate the FAdV-4C DNA standard curve, the target sequence located between $1293 \mathrm{nt}$ to $1417 \mathrm{nt}$ of hexon gene was cloned into the pGEM-T easy vector to create a pGEM-T-hexon plasmid. Copy numbers of the viral DNAs in the selected tissue specimens were calculated by comparison of the standard curve based on the positive template of the pGEM-T-hexon plasmid as previously described $[26,27]$.

\section{Real-time RT-PCR for quantification of cytokine mRNA levels}

Real-time qRT-PCR assay for tissues was used as previously described [28, 29]. Briefly, approximately $100 \mathrm{mg}$ of tissues were diluted with $1 \mathrm{~mL}$ sterilized PBS then homogenized at a speed of $7000 \times \mathrm{g}$ for $2 \mathrm{~min}$ by TissueLyser-PEII apparatus (Jingxin, Shanghai, China). After centrifugation at $10,000 \times g$ for $5 \mathrm{~min}, 140 \mu \mathrm{L}$ aliquots of the supernatants were collected for RNA extraction with RNeasy Mini kit (Qiagen), then the mRNA was used for cDNA synthesis with Oligo (dT)18 (Promega Co., Madison, WS, USA). Primers of the $\beta$-actin and pro-inflammatory cytokine genes (Additional file 1: Table S2) were used for real-time qRT-PCR analysis with AceQ $^{\circ}$ qPCR SYBR ${ }^{\circledR}$ Green Master Mix (Vazyme Inc., Nanjing, China) according to the following cycle protocol: $95^{\circ} \mathrm{C}$ for $5 \mathrm{~min}, 40 \mathrm{cycles}$ at $95^{\circ} \mathrm{C}$ for $10 \mathrm{~s}(\mathrm{~s})$ and $60{ }^{\circ} \mathrm{C}$ for $30 \mathrm{~s}$, or followed by the melt curve stage: $95^{\circ} \mathrm{C}$ for $15 \mathrm{~s}$, $60{ }^{\circ} \mathrm{C}$ for $1 \mathrm{~min}$, and $95^{\circ} \mathrm{C}$ for $15 \mathrm{~s}$.

\section{Immunohistochemistry assay}

Tissues were fixed in $10 \%$ neutral formalin buffer, dehydrated, embedded, and then sectioned for Hematoxylin \& Eosin ( $\mathrm{H} \& \mathrm{E})$ staining. The immunohistochemistry (IHC) assay of the liver and kidney slides with typical lesions was carried out as previously described [30]. Briefly, the IHC analysis was performed with monoclonal antibody (mAb) 1B4 specific for the detection of the Hexon protein using the avidin-biotin-peroxidase, which was prepared in the lab. Tissue sections were incubated with the primary mAb $1 B 4(1: 1,000)$ at $4{ }^{\circ} \mathrm{C}$ for $6 \mathrm{~h}$ and next blocked with $2 \%$ BSA for $1 \mathrm{~h}$ and then incubated with the biotinylated secondary antibody and avidinbiotin-peroxidase complex (Boster, Wuhan, China) (1:2, 000). Representative images were caught under a Nikon microscope with an Olympus DP25 camera.

\section{Statistical analysis}

Results are presented as means \pm standard errors of the mean. All the data were graphed and statistical analyses were performed using Prism 7 software (GraphPad, La Jolla, CA). Two groups' means were compared with a paired two-tailed student $t$ test, whereas multiple comparisons were carried out using analysis of variance (one-way ANOVA method). The differences were considered statistically significant at $p$ values of $<0.01$ or $<0.05$.

\section{Supplementary information}

Supplementary information accompanies this paper at https://doi.org/10. 1186/s12917-019-2092-5.

Additional file 1: Table S1. Primers used to amplify the complete genomic sequence of FAdV-C4V. Table S2. Primers for PCR identification and quantitation by Real-time PCR. Table S3. Comparison of variable amino acid sequences from Fiber-2 among HPS and non-HPS isolates.

\section{Abbreviations \\ bp: base pairs; CEK: Primary chicken embryo kidney; CPE: Cytopathic effects; Ct: Threshold cycle; dpc: day post-challenge; ELD 50 : 50\% egg lethal dose; FAdV-4: FAdV serotype 4; H \& E: Hematoxylin \& Eosin; hpi: hours' post- inoculation; HPS: Hydropericardium syndrome; IBH: Inclusion body hepatitis; IHC: Immunohistochemistry; LMH: Leghorn male hepatoma chicken liver; mAb: monoclonal antibody; $\mathrm{OsO}_{4}$ : Osmium tetroxide; PBS: Phosphate- buffered saline; PCR: Polymerase chain reaction; RT: Reverse transcription; $\mathrm{TCID}_{50}: 50 \%$ tissue culture infection doses; Th1: T helper 1 cells}

\section{Acknowledgements}

Not Applicable.

\section{Authors' contributions}

Conceived and designed the experiments: HC, JY, KW, and HS. Performed the experiments: KW, HS, ZY, and YL. Analyzed the data: HC, JY, KW, and HS. Contributed reagents/materials/analysis tools: HC. Wrote the paper: KW, HS, and HC. All authors have read and approved the final version of the manuscript.

\section{Funding}

This work was supported by Key project for Agriculture from Shanghai Agriculture Commission (201702080008F00068), National Key Research and Development Program of China (2017YFD0500702 and 2017YFD0502302), and the National Natural Science Foundation of China (No. 31572502). Neither the design of the study, nor the collection, analysis, and interpretation of data and writing the manuscript was influenced by these funding sources. The funding agencies did not participate in study design, data collection, analysis and interpretation or writing of the manuscript.

\section{Availability of data and materials}

The datasets used and/or analyzed during the current study are available from the corresponding author on reasonable request.

\section{Ethics approval and consent to participate}

The study was submitted to and approved by Shanghai Veterinary Research Institutional Ethics Committee and a statement to this effect was included with an ID number as SHVRI-PO-2016-0276. 


\section{Competing interests}

The authors declare that they have no competing interests.

\section{Author details}

'Shanghai Veterinary Research Institute, CAAS, Shanghai 200241, China. ${ }^{2}$ College of Veterinary Medicine, Yangzhou University, Yangzhou 225009, China.

Received: 4 October 2018 Accepted: 16 September 2019

Published online: 28 October 2019

\section{References}

1. Hess M. Detection and differentiation of avian adenoviruses: a review. Avian Pathol. 2000;29(3):195-206.

2. Meulemans G, Boschmans M, Berg TP, Decaesstecker M. Polymerase chain reaction combined with restriction enzyme analysis for detection and differentiation of fowl adenoviruses. Avian Pathol. 2001;30(6):655-60.

3. Shah MA, Ullah R, March M, Shah MS, Ismat F, Habib M, lqbal M, Onesti S, Rahman M. Overexpression and characterization of the $100 \mathrm{~K}$ protein of fowl adenovirus-4 as an antiviral target. Virus Res. 2017:238:218-25.

4. Shah MS, Ashraf A, Khan MI, Rahman M, Habib M, Chughtai MI, Qureshi JA. Fowl adenovirus: history, emergence, biology and development of a vaccine against hydropericardium syndrome. Arch Virol. 2017;162(7):1833-43.

5. Gunes A, Marek A, Grafl B, Berger E, Hess M. Real-time PCR assay for universal detection and quantitation of all five species of fowl adenoviruses (FAdV-A to FAdV-E). J Virol Methods. 2012:183(2):147-53.

6. Pan Q, Liu L, Gao Y, Liu C, Qi X, Zhang Y, Wang Y, Li K, Gao L, Wang X, et al. Characterization of a hypervirulent fowl adenovirus 4 with the nove genotype newly prevalent in China and establishment of reproduction infection model of hydropericardium syndrome in chickens. Poult Sci. 2017; 96(6):1581-8.

7. McFerran JB, Smyth JA. Avian adenoviruses. Rev Sci Tech. 2000;19(2):589-601.

8. Gjevre AG, Kaldhusdal M, Eriksen GS. Gizzard erosion and ulceration syndrome in chickens and turkeys: a review of causal or predisposing factors. Avian Pathol. 2013:42(4):297-303.

9. Niu Y, Sun Q, Zhang G, Sun W, Liu X, Xiao Y, Shang Y, Liu S. Epidemiological investigation of outbreaks of fowl adenovirus infections in commercial chickens in China. Transbound Emerg Dis. 2018;65(1):e121-6.

10. Ganesh K, Raghavan R, Gowda RN, Satyanarayana ML, Suryanarayana W. Purification and characterization of the aetiological agent of hydropericardium hepatitis syndrome from infected liver tissues of broiler chickens. Trop Anim Health Prod. 2002;34(1):7-17.

11. Choi KS, Kye SJ, Kim JY, Jeon WJ, Lee EK, Park KY, Sung HW. Epidemiological investigation of outbreaks of fowl adenovirus infection in commercial chickens in Korea. Poult Sci. 2012;91(10):2502-6.

12. Pan Q, Yang Y, Shi Z, Liu L, Gao Y, Qi X, Liu C, Zhang Y, Cui H, Wang X. Different dynamic distribution in chickens and ducks of the Hypervirulent, novel genotype fowl adenovirus serotype 4 recently emerged in China. Front Microbiol. 2017;8:1005.

13. Ye J, Liang G, Zhang J, Wang W, Song N, Wang P, Zheng W, Xie Q, Shao H, Wan Z, et al. Outbreaks of serotype 4 fowl adenovirus with novel genotype, China. Emerg Microbes Infect. 2016;5:e50.

14. Liu Y, Wan W, Gao D, Li Y, Yang X, Liu H, Yao H, Chen L, Wang C, Zhao J. Genetic characterization of novel fowl aviadenovirus 4 isolates from outbreaks of hepatitis-hydropericardium syndrome in broiler chickens in China. Emerg Microbes Infect. 2016;5(11):e117.

15. Wang K, Cai X, Ye J, Liu H, Zhang Q, Liu Q, Li Z, Chen H. Isolation and characterization of group I Fowl adenovirus serotype 4 variant strain of inclusion body hepatitis virus in chickens \%. Chinese J Animal Infect Dis. 2016;24(04):1-6.

16. Pan Q, Yang Y, Gao Y, Qi X, Liu C, Zhang Y, Cui H, Wang X. An inactivated Novel Genotype Fowl Adenovirus 4 Protects Chickens against the Hydropericardium Syndrome That Recently Emerged in China. Viruses. 2017:9(8):216.

17. Niu Y, Sun Q, Zhu M, Zhao J, Zhang G, Liu X, Xiao Y, Liu S. Molecular epidemiology and phylogenetic analysis of fowl adenoviruses caused hydropericardium outbreak in China during 2015. Poult Sci. 2018;97(3):803-11.

18. Zhao J, Zhong Q, Zhao Y, Hu YX, Zhang GZ. Pathogenicity and complete genome characterization of fowl adenoviruses isolated from chickens associated with inclusion body hepatitis and Hydropericardium syndrome in China. PLoS One. 2015;10(7):e0133073.
19. Li R, Li G, Lin J, Han S, Hou X, Weng H, Guo M, Lu Z, Li N, Shang Y, et al. Fowl adenovirus serotype 4 SD0828 infections causes high mortality rate and cytokine levels in specific pathogen-free chickens compared to ducks. Front Immunol. 2018:9:49.

20. Grgic H, Poljak Z, Sharif S, Nagy E. Pathogenicity and cytokine gene expression pattern of a serotype 4 fowl adenovirus isolate. PLoS One. 2013:8(10):e77601.

21. Sun JH, Lu P. The potential of avian cytokines as immunotherapeutics and vaccine adjuvants. Sheng Wu Gong Cheng Xue Bao. 2003;19(2):141-6.

22. Sarfraz M, Suleman M, Tikoo SK, Wheler C, Potter AA, Gerdts V, Dar A. Immune responses to in ovo vaccine formulations containing inactivated fowl adenovirus $8 \mathrm{~b}$ with poly [di (sodium

carboxylatoethylphenoxy)]phosphazene (PCEP) and avian beta defensin as adjuvants in chickens. Vaccine. 2017;35(6):981-6.

23. Wu Z, Hu T, Rothwell L, Vervelde L, Kaiser P, Boulton K, Nolan MJ, Tomley FM, Blake DP, Hume DA. Analysis of the function of IL-10 in chickens using specific neutralising antibodies and a sensitive capture ELISA. Dev Comp Immunol. 2016;63:206-12.

24. Pizzi M. Sampling variation of the fifty percent end-point, determined by the reed-Muench (Behrens) method. Hum Biol. 1950;22(3):151-90.

25. Chhabra R, Kuchipudi SV, Chantrey J, Ganapathy K. Pathogenicity and tissue tropism of infectious bronchitis virus is associated with elevated apoptosis and innate immune responses. Virology. 2016:488:232-41.

26. Marek A, Kajan GL, Kosiol C, Benko M, Schachner A, Hess M. Genetic diversity of species fowl aviadenovirus D and fowl aviadenovirus E. J Gen Virol. 2016;97(9):2323-32.

27. Kajan GL, Davison AJ, Palya V, Harrach B, Benko M. Genome sequence of a waterfowl aviadenovirus, goose adenovirus 4. J Gen Virol. 2012;93(Pt 11):2457-65.

28. Huang Q, Wang K, Pan L, Qi K, Liu H, Chen H. Co-infection of H9N2 subtype avian influenza virus and infectious bronchitis virus decreases SP-A expression level in chickens. Vet Microbiol. 2017;203:110-6.

29. Sun H, Yao W, Wang K, Qian Y, Chen H, Jung YS. Inhibition of neddylation pathway represses influenza virus replication and pro-inflammatory responses. Virology. 2018;514:230-9.

30. Chen H, Angel M, Li W, Finch C, Gonzalez AS, Sutton T, Santos J, Perez DR. All-in-one bacmids: an efficient reverse genetics strategy for influenza a virus vaccines. J Virol. 2014;88(17):10013-25.

\section{Publisher's Note}

Springer Nature remains neutral with regard to jurisdictional claims in published maps and institutional affiliations.
Ready to submit your research? Choose BMC and benefit from:

- fast, convenient online submission

- thorough peer review by experienced researchers in your field

- rapid publication on acceptance

- support for research data, including large and complex data types

- gold Open Access which fosters wider collaboration and increased citations

- maximum visibility for your research: over $100 \mathrm{M}$ website views per year

At $\mathrm{BMC}$, research is always in progress.

Learn more biomedcentral.com/submissions 